\title{
Aerosol particle transport and deposition in a CT-scan based mouth-throat model
}

Cite as: AIP Conference Proceedings 2121, 040011 (2019); https://doi.org/10.1063/1.5115882

Published Online: 18 July 2019

S. C. Saha, M. S. Islam, M. Rahimi-Gorji, and M. M. Molla

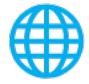

\section{Conference Proceedings}

Get $30 \%$ off all print proceedings!

\section{Enter Promotion Code PDF30 at checkout}

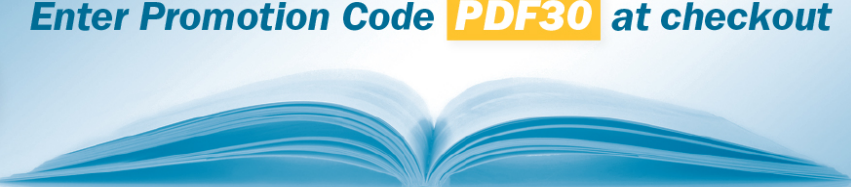




\title{
Aerosol Particle Transport and Deposition in a CT-Scan Based Mouth-Throat Model
}

\author{
S. C. Saha ${ }^{1 *}$, M. S. Islam ${ }^{1}$, M. Rahimi-Gorji ${ }^{2,3}$ and M.M. Molla ${ }^{4}$ \\ ${ }^{1}$ School of Mechanical and Mechatronics Engineering \\ University of Technology Sydney, Sydney, NSW-2007, Australia \\ ${ }^{2}$ Department of Surgery, Ghent University, Ghent, Belgium \\ ${ }^{3}$ Tissue and Solid Mechanics for Medical Applications Lab, Ghent University, Belgium \\ ${ }^{4}$ Department of Mathematics and Physics, North South University, Dhaka-1229, Bangladesh \\ *Corresponding author: suvash.saha@uts.edu.au
}

\begin{abstract}
A precise understanding of the aerosol particle transport and deposition (TD) in the realistic mouth-throat model is important for the respiratory health risk assessment and effective delivery of the aerosol medicine to the targeted positions of the lung. A wide range of studies have developed the particle TD framework for both idealized and non-idealized extra-thoracic airways. However, all of the existing in silico and experimental model reports a significant amount of aerosol particles are deposit at the extra-thoracic airways and the existing drug delivery device can deliver only 12 percent of the aerosol drug to the targeted position of the lung. This study aims to increase the efficiency of the targeted drug delivery by developing a realistic particle transport model for CT-Scan based mouththroat replica. A 3-D realistic mouth-throat model is developed from the CT-Scan DiCom images of a healthy adult cast. High-Quality computational cells are generated for the replica model and the proper grid refinement test has been performed. ANSYS Fluent (19.1) solver is used for the particle TD computation. Tecplot and MATLAB software are used for the post-processing purpose. The numerical results report that the breathing pattern and particle diameter influences the overall particle TD in the mouth-throat model. The numerical results also depict different deposition hot spots for the mouth-throat model, which will eventually help to design a better drug delivery device. The numerical results reported that only 13.67 percent of the $10-\mu \mathrm{m}$ diameter particles are deposited at the mouth-throat model at 15 lpm flow rate and which indicate that the remaining particles will move to the beyond airways. The present results along with more case studies will develop the understanding of the realistic particle deposition in the extrathoracic airways.
\end{abstract}

\section{INTRODUCTION}

Inhalation and exhalation processes have attracted considerable attention among researchers in recent years in response to different respiratory diseases that relate to the lung disorder. The air exchange procedure in the case of breathing, coughing, and sneezing plays an important role in biomedical and biomechanical engineering. From the last century, researchers have been using different approaches for better understanding of the acoustic impedance process of the human respiratory system. Understanding of particulate matter (PM) transportation and deposition in the airways is the primary step in predicting and preventing respiratory diseases. Different natural and man-made sources emit a significant amount of suspended particles to the atmospheric air. These suspended particles consist of a complex size distribution, which determines the deposition location in the respiratory airways ${ }^{1}$. Different deposition mechanisms (inertial impaction, Brownian diffusion, gravitational sedimentation, and interception) guide the deposition pattern in the human lung ${ }^{2}$. Larger diameter particles mostly deposit at the extrathoracic airways of the human lung ${ }^{3}$. Human lung extrathoracic airways (nasal cavities, mouth-throat) is the primary gateway of the atmospheric aerosol transport and deposition to the lung airways. A precise knowledge of the airflow and particle transport at the extrathoracic airways of the human lung is important for the better health risk assessment. A wide range in vivo and in silico model ${ }^{4-12}$ have conducted the aerosol particle transport and deposition in the human respiratory airways. Almost all of the published literature ${ }^{13-15}$ consider non-realistic anatomical model for the extrathoracic airways.

Aerosol particle transport and flow analysis for an idealized mouth-throat model show the Reynolds number influence the overall deposition ${ }^{13}$. The gamma scintigraphy and gravimetry study illustrate that the deposition efficiency increases with the Reynolds number even if the stokes numbers remain constant. A number of studies for

8th BSME International Conference on Thermal Engineering

AIP Conf. Proc. 2121, 040011-1-040011-6; https://doi.org/10.1063/1.5115882

Published by AIP Publishing. 978-0-7354-1861-5/\$30.00 
radioactive aerosol transport and deposition have been performed for mouth-throat model ${ }^{16,17}$. All of those studies calculate the overall deposition from the measured activities. Matidaet al. performed a numerical calculation for monodisperse particle transport and deposition in an idealized mouth-throat model ${ }^{18}$. A more detail experimental and numerical study of the highly idealized mouth-throat model investigates the microparticle deposition and the total deposition from the numerical study shows that turbulence mixing plays a negligible role in the particle deposition ${ }^{15}$. The total and localized deposition pattern from the numerical study shows approximately $20 \%$ variation of deposition with the measurement data. However, it is difficult to understand the flow dynamics and particle deposition pattern at the extrathoracic region because of the variation of the morphological variability and sophisticated inhalation pattern. Moreover, the idealized anatomical model of extrathoracic airways is far from the realistic anatomical model. The understanding of the pharmaceutical particle transport and deposition in the mouththroat model is important for the targeted drug delivery system as most of the drug particles are deposit in this region. The proper knowledge of the aerosol particle transport and deposition in a realistic mouth-throat model is also essential for better respiratory health assessment and targeted drug delivery system. This study will investigate aerosol particle transport and deposition in CT-Scan based realistic mouth-throat model.

\section{METHODS}

The anatomical structure of the mouth-throat model is generated from the CT-Scan Di-Com images of a healthy adult. The medical image processing software AMIRA is used to convert the DiCom image to the solid 3D model. The Geomagic software is used for the surface reconstruction and a 3-D mouth-throat model is developed. Ansys meshing module is used to construct the computational grid for the extrathoracic airways (see figure 1). The unstructured tetrahedral cells aregenerated for the mouth-throat model. A ten layer dense hexahedral inflation elements aregenerated to capture the complex flow behavior near to the lung surface. The transition ratio value of 0.272 was used to create the inflation layer near the surface.Figure 1(a) shows the generated tetrahedral elements and figure 1(b) shows the cross-sectional view of the inflation layer mesh. The mesh refinement for the mouth-throat model has been performed and the final model contains 0.51 million computational cells.Large Eddy Simulation (LES) turbulent model is used to solve the fluid flow and particle transport in the upper airways. The air is treated as the continuous phase and the particle is treated as the disperse phase.Euler-Lagrange particle tracking scheme is used to investigate the particle transport and deposition in the central airway. ANSYS 17.2 (FLUENT) solver based Discrete Phase Model (DPM) is used to monitor the aerosol particle impact on the geometry wall. Pressure-velocity coupling scheme, SIMPLE is used to solve the DPM particle movement. Second order pressure and bounded central differencing momentum spatial discretization are used for the LES model. A hybrid initialization technique is used to initialize the model. The density of the particle is used as $1100 \mathrm{~kg} / \mathrm{m}^{3}{ }^{19}$. The particles are injected from the inlet surface of the mouth-throat model and all particles are injected at once. The particles are injected from the normal direction of the inlet surface and spherical drag law is used to define the particle shape. The velocity inlet and pressure outlet boundary conditions are used to address the equation of flow ${ }^{20,21}$. The wall boundary condition is set as a trap to investigate the particle impact on the wall. The numerical model has been validated with the available published literature and it can be found in the authors previous study ${ }^{22}$.
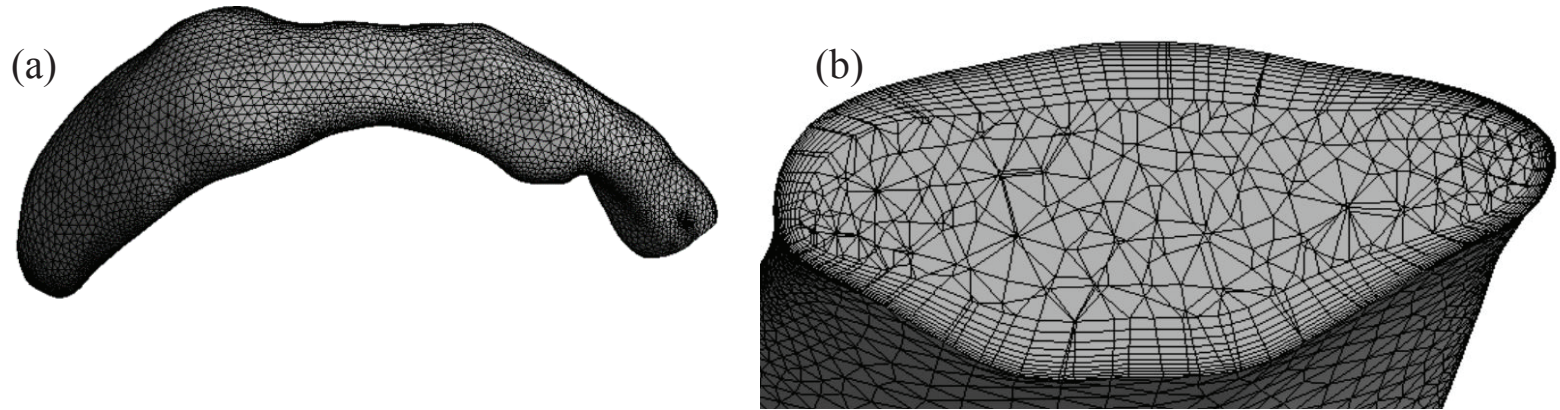

FIGURE 1.Computational mesh for the mouth-throat model, (a) unstructured tetrahedral elements for the whole mouth-throat model and (b) cross-sectional view of the inflation layer mesh. 


\section{RESULTS AND DISCUSSIONS}

The current study accounts for spherical micro-particle transport and deposition in the CT-based mouth-throat model. The present numerical model has extensively investigated the size specific monodisperse particle transport and deposition at $15 \mathrm{lpm}$ and $30 \mathrm{lpm}$ flow rate. Monodisperse particles diameter of $1-\mu \mathrm{m}, 5-\mu \mathrm{m}$, and $10-\mu \mathrm{m}$ are used to study the particle size effects on deposition. Figure 2 shows the velocity contours and velocity vectors at $15 \mathrm{lpm}$ flow rate. The velocity and vector contours are drawn at the selected planes for the mouth-throat model. The velocity contours at plane A and plane B show a complex flow pattern at the upper part of the mouth-throat. The anatomical structure of the mouth-throat model and centrifugally induced pressure gradient creates the dean vortex at the mouth-throat region. The vector contours at different planeillustrate the flow directions. The velocity and vector contours at the larynx (plane D and plane E) region also show a complicated flow pattern and creates the Dean vortices.

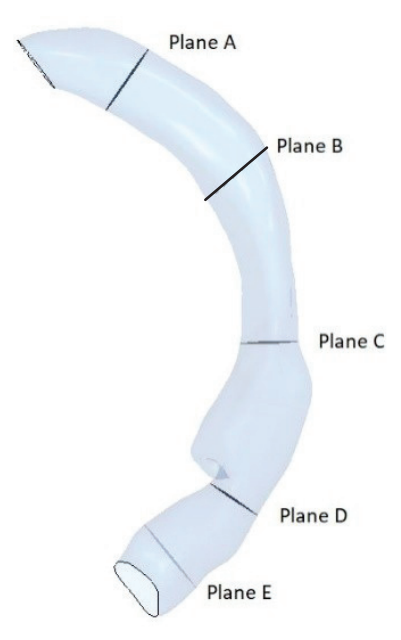

(A)
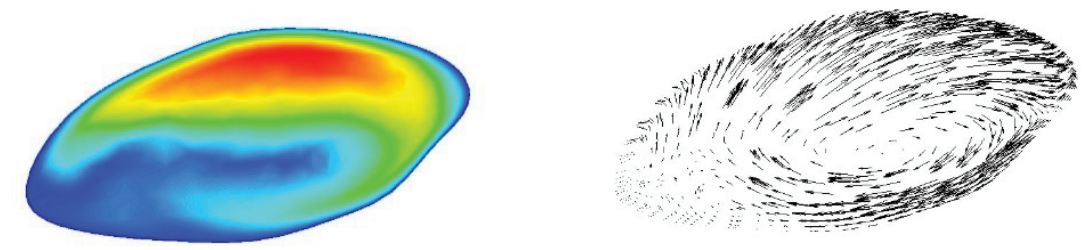

(B)
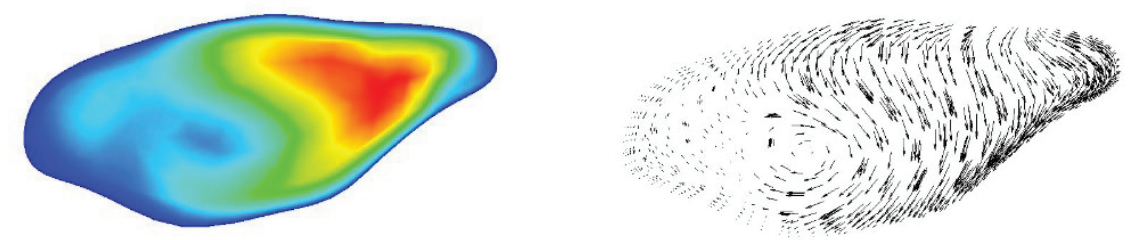

(C)
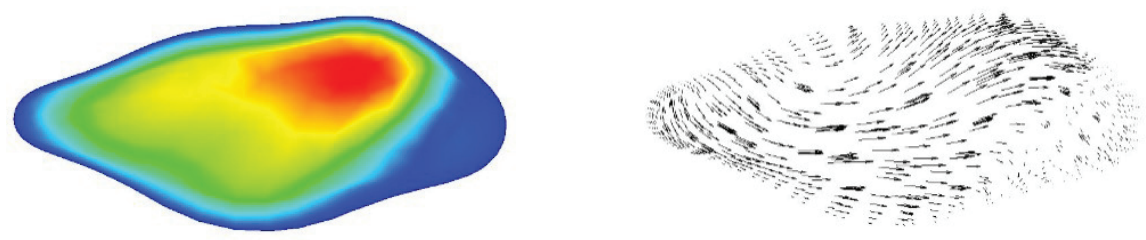

(D)
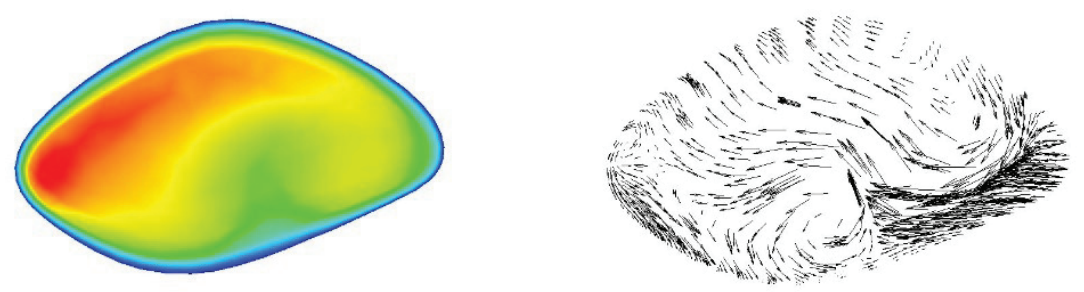

(E)
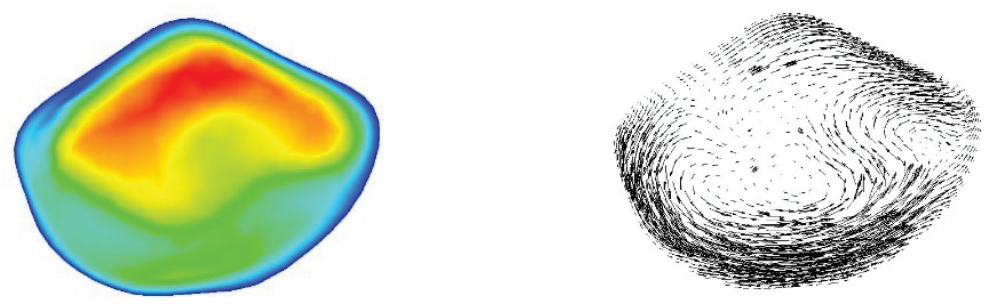

FIGURE 2.Velocity and vector contour at the selected planes of the mouth-throat model. 
The velocity profiles are depicted at the selected positions on the mouth-throat model for $15 \mathrm{lpm}$ and $30 \mathrm{lpm}$ flow rates. Figure 3 shows the velocity profiles at four different positions on the mouth-throat model. The overall velocity profile for 30 lpmflowrate shows a large gradient at the selected positions than the $15 \mathrm{lpm}$ flow rate. At 15 lpm flow rate, the velocity profile at position 1 and 2 shows a similar velocity pattern as the fluid flow at a mouththroat region at less than $30 \mathrm{lpm}$ is mostly laminar ${ }^{23}$. The velocity profile at position D for $15 \mathrm{lpm}$ and $30 \mathrm{lpm}$ flow rate shows a more complicated flow pattern compare to the other positions.

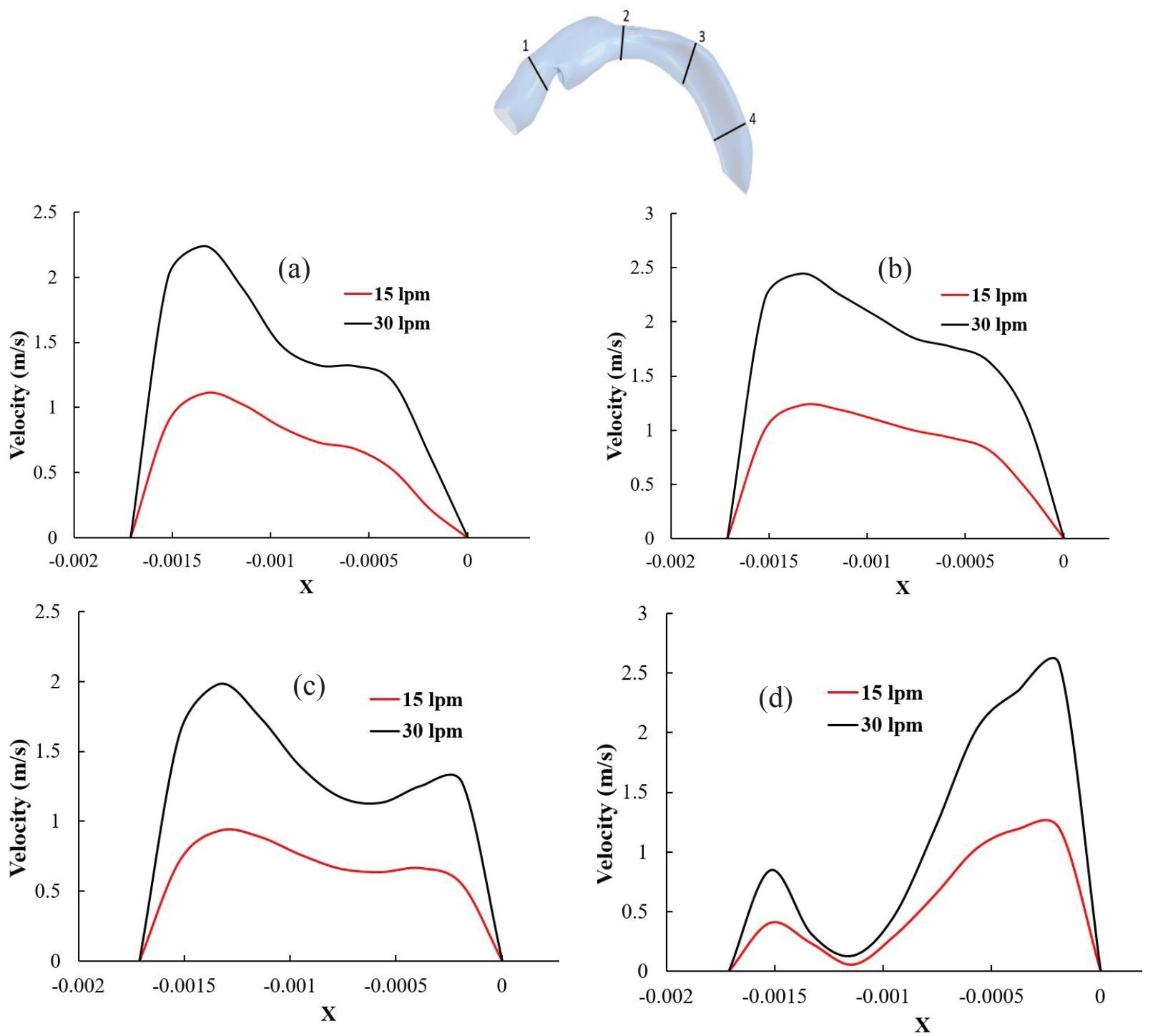

FIGURE 3: Velocity profiles at the selected positions of the mouth-throat model at $15 \mathrm{lpm}$ flow rate, (a) Position 1, (b) position 2, (c) position 3 , and (d) position 4.

The velocity streamlines for the mouth-throat model at $15 \mathrm{lpm}$ and $30 \mathrm{lpm}$ flow rate is calculated. Figure 4(a) shows the velocity streamline for $15 \mathrm{lpm}$ flow rate. The velocity streamline shows a highly complex flow path at the upper part of the mouth-throat model. At $30 \mathrm{lpm}$ flow rate, the velocity streamlines at the glottis and larynx regions show a complex flow path. The fluid flow becomes locally turbulent at the glottis region at the flow rate greater than or equal $30 \mathrm{lpm}$ and the turbulent dispersion may exist to the to the next couple of generations ${ }^{24}$. 

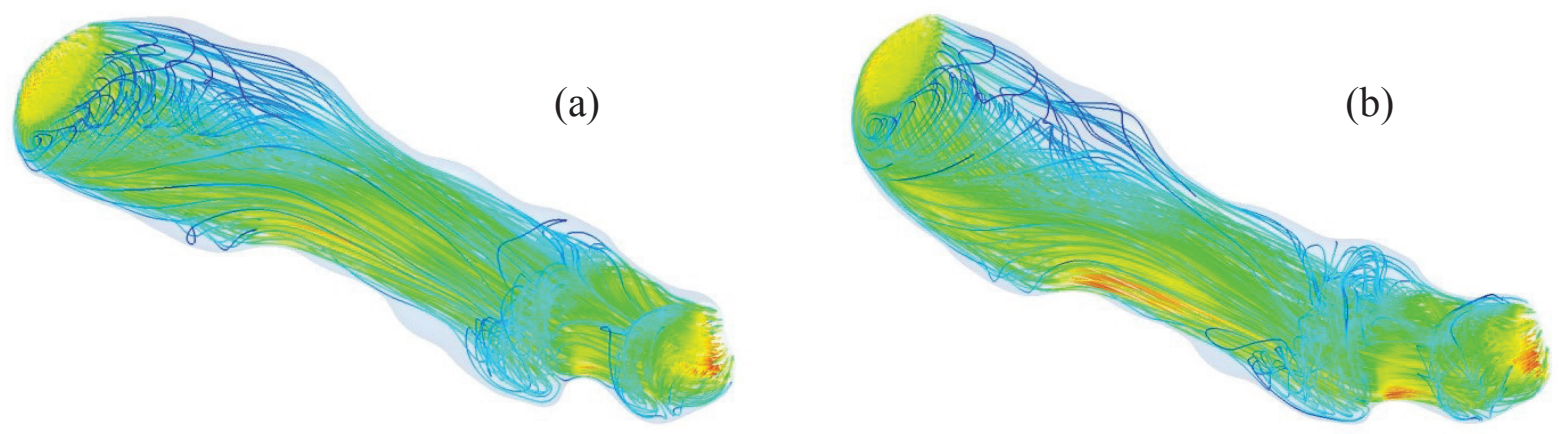

FIGURE 4: Velocity streamlines at the mouth-throat model for (a) $15 \mathrm{lpm}$ flow rate and (b) $30 \mathrm{lpm}$ flow rate.

The aerosol particle deposition pattern at the mouth-throat model is calculated for different flow rates. Figures 5 $(\mathrm{a}, \mathrm{b})$ show the deposition pattern for $1-\mu \mathrm{m}$ and $10-\mu \mathrm{m}$ diameter particles at $15 \mathrm{lpm}$ flow rate. The overall deposition pattern at $15 \mathrm{lpm}$ flow rate shows a significant amount of $1-\mu \mathrm{m}$ and $10-\mu \mathrm{m}$ diameter particles are deposited at the upper part of the mouth-throat model. The deposition pattern at $15 \mathrm{lpm}$ shows no deposition at the larynx region and only a few particles are deposited to the lower region of the mouth-throat. Figures 5(c, d) shows the deposition pattern for $10-\mu \mathrm{m}$ diameter particles at $30 \mathrm{lpm}$ and $90 \mathrm{~lm}$ flow rates. The general deposition pattern reports that a significant amount of particles are deposited for $90 \mathrm{lpm}$ flow rate than the $30 \mathrm{lpm}$ flow rate. At $90 \mathrm{lpm}$ flow rate, a substantial amount of particles are deposited at the larynx region. The higher flow rate, micro-particle inertia, and turbulent dispersion influence the $10-\mu \mathrm{m}$ diameter particle deposition at the larynx regions. The deposition efficiency (DE) of $10-\mu \mathrm{m}$ diameter particles at $90 \mathrm{lpm}$ flow rate is $93.93 \%$ whereas, at $30 \mathrm{lpm}$ flow rate, the DE of $10-\mu \mathrm{m}$ diameter particles is $27.62 \%$.

(a)

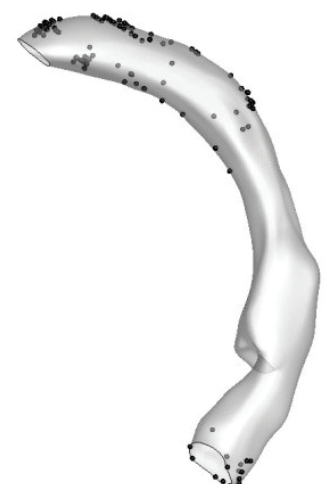

(c)

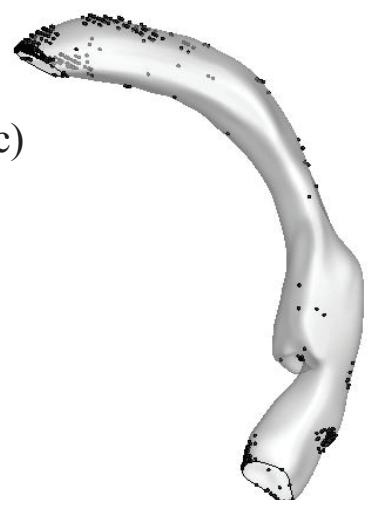

(b)
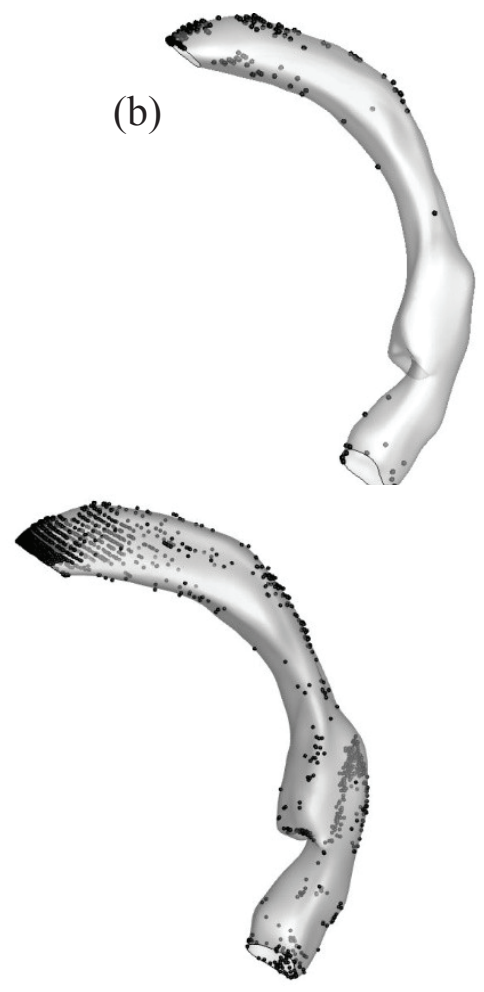

(d)

FIGURE 5: Aerosol particle deposition pattern for (a) $15 \mathrm{lpm}$ flow rate and 1- $\mu \mathrm{m}$ particle (b) $15 \mathrm{lpm}$ flow rate and $10-\mu \mathrm{m}$ particle, (c) $30 \mathrm{lpm}$ flow rate and 10- $\mu \mathrm{m}$ particle and (d) $90 \mathrm{lpm}$ flow rate and 10- $\mu \mathrm{m}$ particle. 


\section{CONCLUSIONS}

The present CFD model investigates the monodisperse particle transport and deposition in a mouth-throat model. The CT-based realistic anatomical model employed an advanced meshing technique to generate the computational cells. A numerical model is developed to predict the spherical micro-particle transport and deposition in the mouththroat model. Different flow rates are considered to investigate the monodisperse micro-particle deposition at the mouth-throat model. The following conclusions can be drawn from the present study:

- Turbulent dispersion influences the spherical micro-particle transport and deposition. The overall deposition concentration increases with the flow rates;

- At lower flow rate, the mouth-throat upper region is the deposition hot spot. On contrary, at a higher flow rate, larynx region is the deposition hotspot;

The findings of the present study and more inclusive polydisperse particle investigation for the mouth-throat model will help the understanding of the drug delivery system to the tracheobronchial airways. The present model can identify the zone-specific deposition hotspot, which could potentially assist the targeted drug delivery system. A comprehensive polydisperse micro- and nanoparticle transport and deposition for the digital airway geometry will be studied in the near future.

\section{REFERENCES}

1. H. Schulz, P. Brand and J. Heyder, Lung biology in health and disease 143, 229-277 (2000).

2. J.-I. Choi and C. S. Kim, Inhalation toxicology 19 (11), 925-939 (2007).

3. M. Everard, Journal of Aerosol Medicine 14 (1, Supplement 1), 59-64 (2001).

4. Islam, S. C. Saha and P. M. Young, 21st Australasian Fluid Mechanics Conference, Adelaide, Australia (2018).

5. M. Rahimi-Gorji, T. B. Gorji and M. Gorji-Bandpy, Computers in biology and medicine 74, $1-17$ (2016).

6. A. V. Kolanjiyil, C. Kleinstreuer and R. T. Sadikot, Computers in biology and medicine 84, 247-253 (2017).

7. M. Nordlund, M. Belka, A. K. Kuczaj, F. Lizal, J. Jedelsky, J. Elcner, M. Jicha, Y. Sauser, S. Le Bouhellec and S. Cosandey, Inhalation Toxicology 29 (3), 113-125 (2017).

8. P. W. Longest, G. Tian, N. Khajeh-Hosseini-Dalasm and M. Hindle, Journal of aerosol medicine and pulmonary drug delivery (2016).

9. M. S. Islam, S. C. Saha, E. Sauret and Y. Gu, 20th Australasian Fluid Mechanics Conference, Perth, Australia 58 December (2016).

10. M. S. Islam, S. C. Saha, E. Sauret, Y. Gu and M. M. Molla, presented at the AIP Conference Proceedings, 2017 (unpublished).

11. M. S. Islam, S. C. Saha, T. Gemci, I. A. Yang, E. Sauret and Y. T. Gu, Scientific Reports 8 (1), 16387 (2018).

12. M. Rahimi-Gorji, O. Pourmehran, M. Gorji-Bandpy and T. Gorji, Journal of Molecular Liquids 209, 121-133 (2015).

13. B. Grgic, W. Finlay and A. Heenan, Journal of Aerosol Science 35 (1), 21-32 (2004).

14. Z. Zhang, C. Kleinstreuer, J. Donohue and C. Kim, Journal of aerosol science 36 (2), 211-233 (2005).

15. Y. Zhang, W. Finlay and E. Matida, Journal of Aerosol Science 35 (7), 789-803 (2004).

16. T. L. Chan and M. Lippmann, The American Industrial Hygiene Association Journal 41 (6), 399-409 (1980).

17. W. Stahlhofen, J. Gebhart and J. Heyder, The American Industrial Hygiene Association Journal 41 (6), 385398a (1980).

18. E. Matida, W. Finlay, C. Lange and B. Grgic, Journal of Aerosol Science 35 (1), 1-19 (2004).

19. A. Virtanen, J. Ristimäki, M. Marjamäki, K. Vaaraslahti, J. Keskinen and M. Lappi, Report No. 0148-7191, 2002.

20. M. S. Islam, S. C. Saha, E. Sauret, T. Gemci, I. A. Yang and Y. Gu, Journal of biomechanics 64, 16-25 (2017).

21. M. S. Islam, S. C. Saha, E. Sauret, Y. Gu and Z. Ristovski, presented at the Proceedings of the International Conference on Computational Methods, 2015 (unpublished).

22. M. S. Islam, S. C. Saha, E. Sauret, T. Gemci and Y. Gu, Journal of Aerosol Science 108, 29-43 (2017).

23. Z. Zhang and C. Kleinstreuer, International Journal for Numerical Methods in Biomedical Engineering 27 (12), 1930-1950 (2011).

24. Z. Zhang and C. Kleinstreuer, Journal of computational physics 198 (1), 178-210 (2004). 